\title{
Retrospective analysis of risk factors of slide positivity among febrile patients in the Salween river valley of Shan Special Region II, northern Myanmar
}

\author{
Hui Liu', Jian-Wei $\mathrm{Xu}^{1 *}$, Qi-Zhang $\mathrm{Xu}^{2}$ and Yi-Rou Zeng ${ }^{3}$
}

\begin{abstract}
Background: In Myanmar, epidemiological conditions have been unclear due to a lack of accurate data. In 2014 and 2016, malaria outbreaks occurred in the Shan Special Region II (SSR2). It was reported that these outbreaks were caused by malaria patients from the Salween River Valley (SRV), but further research is needed to confirm these reports. To examine the risks of malaria infection in the SSR2 section of the SRV, this paper offers a retrospective analysis based on the data we collected in 2009.

Methods: A multivariate logistic model was utilized to analyze risk factors associated with the slide positivity of 2009. Results of the investigation in 2009 were compared with updated data.

Results: The number of slide positivity was 91 (24.7\%, 95\% confidence interval [CI], 20.3-29.4\%) among 369 people who had fever 2 weeks ago of the survey, including $74(20.1 \%$; $95 \% \mathrm{Cl}, 16.1-24.5 \%)$ cases of $P$. falciparum, 13 (3.5\%; $95 \% \mathrm{Cl}$, 1.95.9\%) of P.vivax and $4(1.1 \%, 95 \% \mathrm{Cl}, 0.3-2.8 \%)$ of P. malariae. The adjusted odds ratio (OR) was $99.8(95 \% \mathrm{Cl}, 24.7-887.7)$ for patients' age $<15$ years, $6.61(95 \% \mathrm{Cl}, 3.57-10.49)$ for people living at an altitude of $<800 \mathrm{~m}, 6.35(95 \% \mathrm{Cl}, 2.45-23.27)$ for people lacking knowledge on malaria transmission and knowledge on symptoms, 2.10 (95\% $\mathrm{Cl}, 1.22-5.11)$ for people taking no measures against mosquito bites and $5.55(95 \% \mathrm{Cl}, 2.65-13.05)$ for people delaying treatment. Compared with annual parasitic incidences 13.80 per 10,000 person-years (422/305733) in 2014, 2.36 per 10,000 person-years (73/309004) in 2015 and 5.25 per 10,000 person-years (164/312310) in 2015, malaria burden is reduced.

Conclusion: Age, lower altitude, a lack of knowledge about malaria transmission and symptoms, inaction of measures against mosquito bites and delayed treatment-seeking were independent risk factors for slide positivity. These results indicate that malaria transmission was likely within housing settlements in the SRV, and that the transmission rates within the SRV are higher than in other areas. In order to eliminate malaria, it is important for people to obtain qualified treatment to contain artemisinin resistance.
\end{abstract}

Trial registration: Trial registration number: ChiCTR-COC-17012522.

Retrospectively registered 31 August 2017.

Keywords: Great Mekong Sub-region, Malaria, Risk factor, Salween River Valley, Slide positivity

\footnotetext{
* Correspondence: xjw426@163.com

'Yunnan Institute of Parasitic Diseases, Yunnan Provincial Centre of Malaria

Research, Yunnan Provincial Key Laboratory of Vector-borne Diseases Control

and Research, Yunnan Provincial Collaborative Innovation Center for Public

Health and Disease Prevention and Control, Puer 665000, China

Full list of author information is available at the end of the article
}

(c) The Author(s). 2018 Open Access This article is distributed under the terms of the Creative Commons Attribution 4.0 International License (http://creativecommons.org/licenses/by/4.0/), which permits unrestricted use, distribution, and reproduction in any medium, provided you give appropriate credit to the original author(s) and the source, provide a link to the Creative Commons license, and indicate if changes were made. The Creative Commons Public Domain Dedication waiver (http://creativecommons.org/publicdomain/zero/1.0/) applies to the data made available in this article, unless otherwise stated. 


\section{Background}

Malaria is a disease caused by parasites of the Plasmodium family and is transmitted by female Anopheles mosquitoes. Remarkable progress has been made in malaria control over the past decade, but malaria is still devastatingly affecting human health and livelihoods globally [1]. About 214 million (range 149-303 million) new cases of malaria were identified in 2015, with the number in the South-East Asia Region (10\%) ranking second highest [2]. The Greater Mekong Subregion (GMS) includes Cambodia, Myanmar, Thailand, Vietnam, Laos and Yunnan Province of China. Malaria elimination faces daunting challenges as malaria epidemiology exhibits enormous complexity in the region [3]. Around $70 \%$ of the total population in the GMS is still at risk of contracting malaria and the disease is concentrated mainly in remote areas [4]. Although malaria is well controlled in China and Thailand, a high prevalence of malaria is still seen in Myanmar. In response to the recent emergence of artemisinin-resistant malaria in the GMS, Myanmar has become the main focus in the region. However, further micro epidemiological data from northern Myanmar is needed [5].

The World Health Organization (WHO) plans to eliminate malaria by 2030 in all GMS countries [6]. By 2013, we had reduced the malaria burden significantly along the China-Myanmar border [7-9] However, the report of an outbreak of $P$. falciparum malaria marked the first patient infected with malaria in a private rubber plantation importing from the Salween River Valley, Shan Special Region II (SSR2) in June 2014 [10]. Some other malaria outbreaks were reported in 2016 as well (not published). Meanwhile, Department of Health of SSRII registered that the total number of confirmed malaria cases in 2014, 2015 and 2016 were 442, 73, and 164 respectively. From above evidence, we infer that the Salween River Valley may still be one of the current malaria foci. To support this claim, this manuscript presents the results of slide positivity and associated factors before the intensive interventions of the Global Fund to fight AIDS, Tuberculosis and Malaria (GFATM) program. It then discusses implications of the findings for contemporary malaria control and elimination in the GMS.

\section{Methods}

\section{Study site}

The SSR2 is known locally as Wa State and the main population are of Wa ethnicity. In the SSR2, malaria (An. minimus is the principal vector) is able to be transmited throughout the year with a peak during the later rainy season from September to November [11]. Gelongba and Mandong are two collective settlements in the Salween river valley, SSR2 (Fig. 1). All the health facilities during the investigation are located in Gelongba and Mandong, including a community health centre, two NGO health posts by Aide Medical International (AMI) and two private clinics.

\section{Sampling and investigation}

Based on malaria endemicity and prevalence in the SSR2, we specifically conducted an active detection at the two settlements from October 1st to December 31st, 2009. We sampled 64 villages, with an approximate total population of 18,940. All those who had a self-reported fever in the previous 2 weeks were enrolled in the study after obtaining informed consent [12].

Thick and thin blood smears were prepared for each participant. Two expert microscopists stained malaria blood films with Giemsa and examined by microscopy later. Data on risk factors was collected by interviewing each household head face-to-face with a structured questionnaire (in Chinese). One researcher (Zeng) who could understand both Wa and Chinese Language conducted the interviews in Wa language and filled out the questionnaires in Chinese [12]. Together with the microscopy-diagnosis results, we recorded details of a range of factors contributing to the patient's risk. This included information concerning age and gender of febrile patients, altitude of their house location, family size, family decisions, annual average cash income, malaria knowledge of household heads, use of any measures against mosquito bites, distance from health facilities and time between onset of illness and seeking treatment.

Each Plasmodium falciparum positive individual was treated by administering Dihydroartemisinin-piperaquine tablets (40 mg base dihydroartemisinin and $320 \mathrm{mg}$ piperaquine phosphate per tablet). DAPQ was given once a day for 3 days, based on the recommendation of Ministry of Health, China [13]. Plasmodium vivax objects were treated by Chloroquine (CQ) and primaquine (PQ). CQ was administered once a day for 3 days with a total target dose of $24 \mathrm{mg}$ base $/ \mathrm{kg}$ and PQ was once a day for 8 days with a dose of $0.45 \mathrm{mg}$ base $/ \mathrm{kg} /$ day $[13,14]$.

\section{Data management and analysis}

We checked the completeness of the data and then entered and validated them in EpiData (version 3.1). Epitable Calculator of EPI6.04 was used to calculate the slide positivity rates and binomial 95\% confidence intervals (CI). A multivariate logistic model was used to calculate odds ratios for risk factors associated with the slide positivity. In the model, we chose the results of microscopy as the outcome (dependent) variable. We then chose sex, age, income, family size, altitude, malaria knowledge, mosquito prevention action, family decision, distance from health facilities and treatment seeking as independent variables [12]. Based on a comprehensive analysis of landform and vegetation, an altitude distribution $(<800 \mathrm{~m} ; 800-1200 \mathrm{~m}$, $>1200 \mathrm{~m}$ ) was established for the study site: a) areas of $<$ 


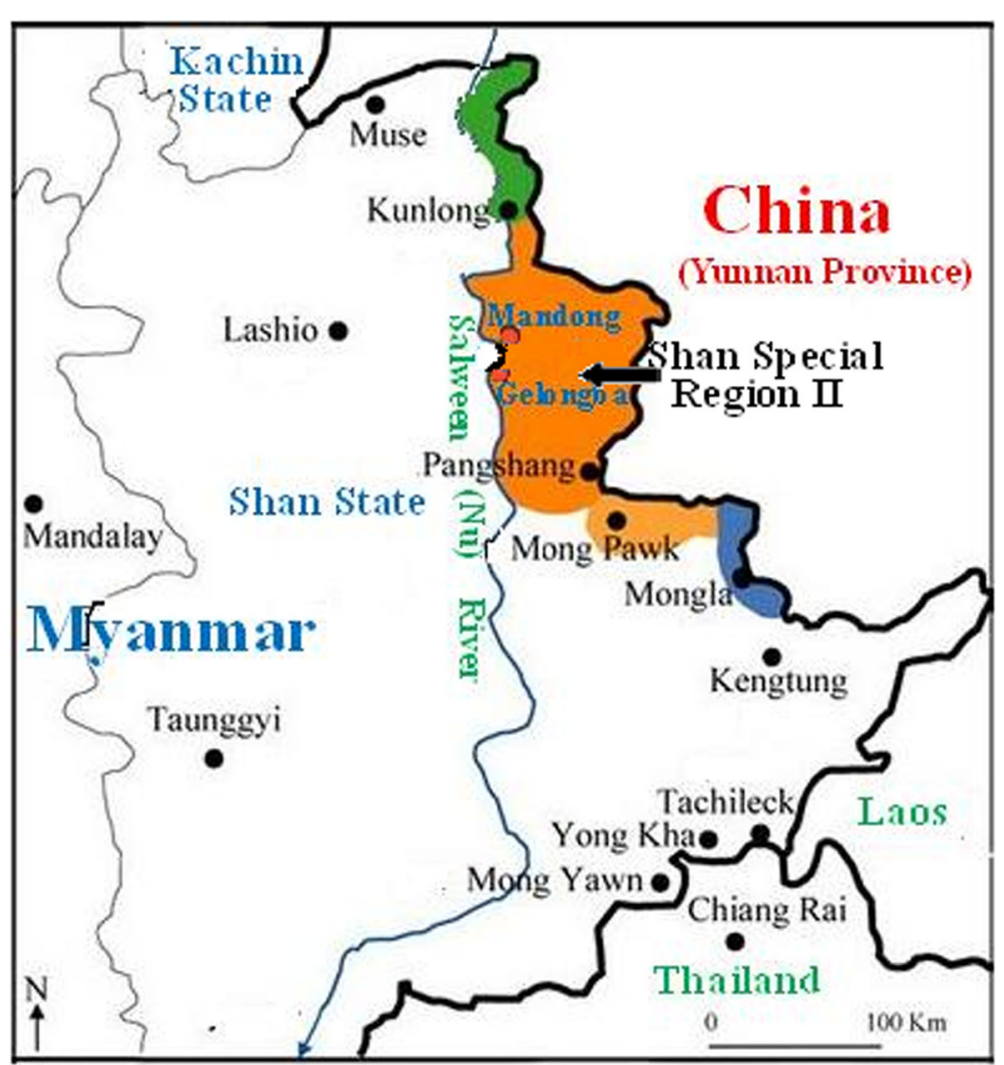

Fig. 1 Study site relative to neighboring areas

$800 \mathrm{~m}$ are usually foothills, most of which are covered by rubber plantations; b) areas 800 to $1200 \mathrm{~m}$ are usually mid hills, most of which are crop (such as dry rice and corn) fields; and areas of $>1200 \mathrm{~m}$ are usually hills, most of which are forested [12]. Finally, the results of the 2009 investigation were compared with the annual parasitic incidences (API) in recent years.

\section{Results}

\section{Subject characteristics}

We visited a total of 718 households and saw 3678 people. After obtaining informed consent, 369 people who had a self-reported fever in the previous 2 weeks were tested by microscopy. The median age of the participants was 23.7 years (range: 3 months - 69 years) and the male/female sex ratio was 1.6 (Table 1 ).

\section{SRV blood positivity, 2009 and SSR2 malaria situation, 2014-2016}

Out of 369 slides prepared in the investigation in 2009, 91 (24.7\%) were positive by microscopy and 74 (81.3\% of positive slides), 13 (14.3\%) and four (4.4\%) of positive slides were $P$. falciparum, P.vivax and $P$. malariae, respectively. The ratio of $P$. falciparum versus $P$. vivax was 5.7. We discovered that a high slide positivity rate and a high proportion of $P$. falciparum might be characteristics of low-altitude regions, especially for children under the age of 5 years who were all parasitic positivity and 10 out of 12 children were infected with P. falciparum. The API were 13.80 per 10,000 person-years (422/ $305733)$ in $2014,2.36$ per 10,000 person-years (73/ $309004)$ in 2015 and 5.25 per 10,000 person-years (164/ 312310 ) in 2016. Additionally, the ratios of $P$. falciparum versus $P$. vivax in 2014, 2015 and 2016 were 1.13 (224/ 198), $0.70(30 / 43)$ and $0.38(45 / 119)$, respectively.

\section{Risk factors for slide positivity}

We identified five independent risk factors associated with slide positivity. First, the slide positivity rate for children (<15 years) was 95.1\% (95\%CI: 83.5-99.4\%). As much as $89.7 \%$ (95\%CI: 75.8-99.1\%) of child positive slides were $P$. falciparum; the adjusted odds ratio (AOR) was 99.8 (95\%CI: 24.7-887.7). Second, people living in low-altitude regions $(<800 \mathrm{~m})$ had significantly higher slide positivity than those living in midaltitude regions ( $\geq 800 \mathrm{~m}$ ) (AOR 6.61[95\%CI: 3.57-10. 49]). Third, those whose household heads who did not know that malaria was transmitted by mosquitoes and had little awareness of the clinical symptoms of malaria had higher slide positivity (AOR 6.35[95\%CI: 
Table 1 Characteristics and slide positivity among febrile patients in the Salween River Valley of Shan Special Region II, Myanmar

\begin{tabular}{|c|c|c|c|c|c|}
\hline & $\begin{array}{l}\text { No. tested } \\
(n=369)\end{array}$ & $\begin{array}{l}\text { No. P. falciparum } \\
(\%, 95 \% \mathrm{Cl})\end{array}$ & $\begin{array}{l}\text { No. P. vivax } \\
(\%, 95 \% \mathrm{Cl})\end{array}$ & $\begin{array}{l}\text { No. P. malariae } \\
(\%, 95 \% \mathrm{Cl})\end{array}$ & $\begin{array}{l}\text { Plasmodium spp. } \\
(\%, 95 \% \mathrm{Cl})\end{array}$ \\
\hline \multicolumn{6}{|l|}{ Demographics } \\
\hline Male & 227 & $37(16.3,11.7-21.8)$ & $10(4.4,2.1-8.0)$ & $3(1.3,0.3-3.8)$ & $50(22.0,18.8-28.0)$ \\
\hline Female & 142 & $37(26.1,19.1-34.1)$ & $3(2.1,0.4-6.0)$ & $1(0.7,0.0-3.9)$ & $41(28.9,21.6-37.1)$ \\
\hline \multicolumn{6}{|l|}{ Age (years) } \\
\hline$<5$ & 12 & $10(83.3,51.6-97.9)$ & $1(8.3,0.2-38.5)$ & $1(8.3,0.2-38.5)$ & $12(100,73.5-100)$ \\
\hline $5-15$ & 29 & $25(86.2,68.3-96.1)$ & $2(6.9,0.8-22.8)$ & $0(0,0-11.9)$ & $27(93.1,77.2-99.2)$ \\
\hline $16-50$ & 276 & $36(13.0,9.3-17.6)$ & $9(3.3,1.5-6.1)$ & $3(1.1,0.2-3.1)$ & $48(17.4,13.1-24.4)$ \\
\hline$>50$ & 52 & $3(5.8,1.2-15.9)$ & $1(1.9,0.1-11.5)$ & $0(0,0-6.8)$ & $4(7.7,2.1-18.5)$ \\
\hline \multicolumn{6}{|l|}{ Annual average cash income (US\$) } \\
\hline$\leq 100$ & 191 & $44(23.0,17.3-29.7)$ & $7(3.7,1.5-7.4)$ & $2(1.0,0.1-3.7)$ & $53(27.7,21.5-34.7)$ \\
\hline $101-200$ & 134 & $26(19.4,13.1-27.1)$ & $5(3.7,1.2-8.5)$ & $1(0.7,0.02-4.1)$ & $32(23.9,16.9-32.0)$ \\
\hline$>200$ & 40 & $4(10.0,2.8-23.7)$ & $1(2.5,0.1-13.2)$ & $1(2.5,0.1-13.2)$ & $6(15.0,5.7-29.8)$ \\
\hline \multicolumn{6}{|l|}{ Family size of the household } \\
\hline$\leq 3$ & 75 & $12(16.0,8.6-26.3)$ & $6(8.0,3.0-16.6)$ & $1(1.3,0.03-7.2)$ & $19(25.3,16.0-36.7)$ \\
\hline $4-5$ & 108 & $33(30.6,22.1-40.2)$ & $5(4.6,1.5-10.5)$ & $2(1.9,0.2-6.5)$ & $40(37.0,27.9-46.9)$ \\
\hline$\geq 6$ & 186 & $29(15.6,10.7-21.6)$ & $2(1.1,0.1-3.8)$ & $1(0.5,0-3.0)$ & $32(17.2,12.1-23.4)$ \\
\hline \multicolumn{6}{|l|}{ Altitude of residence (meters) } \\
\hline$<800$ & 117 & $49(41.9,32.8-51.4)$ & $4(3.4,0.9-8.5)$ & $3(2.6,0.5-7.3)$ & $56(47.9,38.5-57.3)$ \\
\hline $800-1200$ & 143 & $6(4.2,1.6-8.9)$ & $7(4.9,2.0-9.8)$ & $1(0.7,0-3.8)$ & $14(9.9,5.5-16.0)$ \\
\hline$>1200$ & 110 & $19(17.3,10.7-25.7)$ & $2(1.8,0.2-6.4)$ & $0(0,0-3.3)$ & $21(19.1,12.2-27.7)$ \\
\hline \multicolumn{6}{|l|}{ Malaria knowledge of household heads } \\
\hline No malaria knowledge of cause and symptoms & 52 & $17(32.7,20.3-47.1)$ & $5(9.6,3.2-21.0)$ & $0(0,0-6.8)$ & $22(42.3,28.7-56.8)$ \\
\hline Knowing malaria symptoms & 240 & $53(21.1,17.0-27.9)$ & $6(2.5,1.0-5.4)$ & $3(1.3,0.3-3.6)$ & $62(25.8,20.4-31.9)$ \\
\hline Knowing mosquitoes & 77 & $4(5.2,1.4-12.8)$ & $2(2.6,0.3-9.2)$ & $1(1.3,0.04-7.0)$ & $7(9.1,3.7-17.8)$ \\
\hline \multicolumn{6}{|l|}{ Use of measures against mosquito bite } \\
\hline No & 172 & $47(27.3,20.8-34.6)$ & $8(4.6,2.0-9.7)$ & $2(1.2,0.1-4.1)$ & $57(33.1,26.2-40.7)$ \\
\hline Yes & 197 & $27(13.7,9.2-19.3)$ & $5(2.5,0.8-5.8)$ & $2(1.0,0.1-3.6)$ & $34(17.3,12.3-23.3)$ \\
\hline \multicolumn{6}{|l|}{ Family decision } \\
\hline Husband & 283 & $67(23.7,18.8-29.1)$ & $8(2.8,1.2-5.5)$ & $2(0.7,0.1-2.5)$ & $77(27.2,22.1-32.8)$ \\
\hline No response & 11 & $2(18.2,2.3-51.8)$ & $2(18.2,2.3-51.8)$ & $1(9.1,0.2-41.3)$ & $5(45.5,16.7-76.6)$ \\
\hline Wife or co-decision & 75 & $5(6.7,2.1-14.9)$ & $3(4.0,0.8-11.2)$ & $1(1.3,0.03-7.2)$ & $9(12.0,5.6-21.6)$ \\
\hline \multicolumn{6}{|l|}{ Distance from health facilities or drug shops } \\
\hline$>3 \mathrm{~km}$ & 295 & $66(22.4,17.7-27.6)$ & $11(3.7,1.9-6.6)$ & $4(1.4,0.4-3.4)$ & $81(27.5,22.4-32.9)$ \\
\hline$\leq 3 \mathrm{~km}$ & 74 & $8(10.8,4.8-20.2)$ & $2(2.7,0.3-9.4)$ & $0(0,0-4.9)$ & $10(13.5,6.7-23.5)$ \\
\hline \multicolumn{6}{|c|}{ Time between onset of illness and seeking treatment (hours) } \\
\hline Never sought treatment & 46 & $17(37.0,23.2-52.5)$ & $4(8.7,2.4-20.8)$ & $3(6.5,1.4-17.9)$ & $24(52.2,36.9-67.1)$ \\
\hline$>48$ & 181 & $48(26.5,20.2-33.6)$ & $6(3.3,1.2-7.1)$ & $1(0.6,0-3.0)$ & $55(30.4,23.8-37.6)$ \\
\hline $25-48$ & 24 & $2(8.3,1.0-27.0)$ & $2(8.3,1.0-27.0)$ & $0(0,0-14.2)$ & $4(16.7,4.7-37.4)$ \\
\hline$\leq 24$ & 118 & $7(5.9,2.4-11.8)$ & $1(0.8,0.02-4.6)$ & $0(0,0-3.1)$ & $8(6.8,3.0-12.9)$ \\
\hline Total & 369 & $74(20.1,16.1-24.5)$ & $13(3.5,1.9-5.9)$ & $4(1.1,0.3-2.8)$ & $91(24.7,20.3-29.4)$ \\
\hline
\end{tabular}

2.45-23.27]). Fourth, febrile patients who did not use any measures against mosquito bites had higher incidences of malaria infection (AOR 2.10 [95\%CI: 1.22-
5.11]). Finally, people who did not seek treatment within $48 \mathrm{~h}$ had a higher slide positivity rate (AOR5. 55 [95\%CI: 2.65-13.05]) (Table 2). 
Table 2 Risk factors of slide positivity in the Salween River Valley of Shan Special Region II, Myanmar

\begin{tabular}{|c|c|c|c|c|c|}
\hline & $\begin{array}{l}\text { Slide positivity } \\
(\%, 95 \% \mathrm{Cl})\end{array}$ & $\begin{array}{l}\text { Univariate OR } \\
(95 \% \mathrm{Cl})\end{array}$ & $P$ values & $\begin{array}{l}\text { Adjusted OR } \\
(95 \% \mathrm{Cl})\end{array}$ & $P$ values \\
\hline \multicolumn{6}{|l|}{ Sex } \\
\hline Male $(n=227)$ & $50(22.0,18.8-28.0)$ & $0.70(0.42-1.16)$ & 0.1737 & $0.81(0.37-1.26)$ & 0.1936 \\
\hline Female $(n=142)$ & $41(28.9,21.6-37.1)$ & 1 & & 1 & \\
\hline \multicolumn{6}{|l|}{ Age (years) } \\
\hline$<15(n=41)$ & $39(95.1,83.5-99.4)$ & $103.5(25.0-895.8)$ & $<0.0001$ & $99.8(24.7-887.7)$ & $<0.0001$ \\
\hline$\geq 16(n=328)$ & $52(15.8,12.1-20.3)$ & 1 & & 1 & \\
\hline \multicolumn{6}{|l|}{ Annual average income (US\$) } \\
\hline$\leq 100(n=191)$ & $53(27.7,21.5-34.7)$ & $1.37(0.83-2.29)$ & 0.2371 & $1.52(0.81-2.45)$ & 0.2475 \\
\hline$\geq 101(n=174)$ & $38(21.8,15.9-28.7)$ & 1 & & 1 & \\
\hline \multicolumn{6}{|l|}{ Family size of the household } \\
\hline$\leq 5(n=183)$ & $59(32.2,25.5-39.5)$ & $2.29(1.37-3.87)$ & 0.0012 & $0.89(0.67-3.93)$ & 0.1819 \\
\hline$\geq 6(n=186)$ & $32(17.2,12.1-23.4)$ & 1 & & 1 & \\
\hline \multicolumn{6}{|l|}{ Altitude of residence (meters) } \\
\hline$<800(n=117)$ & $56(47.9,38.5-57.3)$ & 5.69 (3.32-9.79) & $<0.0001$ & $6.61(3.57-10.49)$ & $<0.0001$ \\
\hline$\geq 800(n=252)$ & $35(13.9,9.9-18.8)$ & 1 & & 1 & \\
\hline \multicolumn{6}{|l|}{ Malaria knowledge of household heads } \\
\hline No malaria knowledge of cause and symptoms $(n=52)$ & $22(42.3,28.7-56.8)$ & $7.33(2.62-22.22)$ & 0.00002 & $6.35(2.45-23.27)$ & 0.0001 \\
\hline Only knowing malaria symptoms $(n=240)$ & $62(25.8,20.4-31.9)$ & $3.48(1.49-9.43)$ & 0.0033 & $2.98(1.27-10.46)$ & 0.0036 \\
\hline Knowing mosquitoes $(n=77)$ & $7(9.1,3.7-17.8)$ & 1 & & 1 & \\
\hline \multicolumn{6}{|l|}{ Use of measures against mosquito bite } \\
\hline No $(n=172)$ & $57(33.1,26.2-40.7)$ & $2.38(1.42-4.00)$ & 0.0004 & $2.10(1.22-5.11)$ & 0.0015 \\
\hline Yes $(n=197)$ & $34(17.3,12.3-23.3)$ & 1 & & 1 & \\
\hline \multicolumn{6}{|l|}{ Family decision } \\
\hline Husband and no response $(n=294)$ & $82(27.9,22.8-33.4)$ & $2.83(1.32-6.76)$ & 0.0069 & $1.89(0.68-10.77)$ & 0.0765 \\
\hline Wife or co-decision $(n=75)$ & $9(12.0,5.6-21.6)$ & 1 & & 1 & \\
\hline \multicolumn{6}{|l|}{ Distance from health facilities or drug shops } \\
\hline$>3 \mathrm{~km}(n=295)$ & $81(27.5,22.4-32.9)$ & $2.42(1.16-5.54)$ & 0.0152 & $1.62(0.66-8.44)$ & 0.0858 \\
\hline$\leq 3 \mathrm{~km}(n=74)$ & $10(13.5,6.7-23.5)$ & 1 & & 1 & \\
\hline \multicolumn{6}{|l|}{ Time between onset of illness and seeking treatment } \\
\hline$>48 \mathrm{~h}$ and never sought treatment $(n=227)$ & $79(34.8,28.6-41.4)$ & $5.78(2.95-12.15)$ & $<0.0001$ & $5.55(2.65-13.05)$ & $<0.0001$ \\
\hline$\leq 48(n=142)$ & $12(8.5,4.4-14.3)$ & 1 & & 1 & \\
\hline
\end{tabular}

$n$ number tested, $95 \% \mathrm{Cl} 95 \%$ confidence interval, $O R$ odds ratio

\section{Discussion}

Border malaria control is still one of the great challenges in the GMS [3-6]. Reducing malaria endemicity of neighbouring countries largely determines the success of malaria elimination [15-17]. Thus, this paper presents slide positivity and associated risk factors before intensive interventions from China's GFATM in 2009. The slide positivity rate of febrile patients was $24.7 \%(91 / 369)$ and the ratio of $P$. falciparum versus $P$. vivax was 5.7 (74/13). Additionally, a similar active case detection indicated that the malaria prevalence was even higher in former years. The slide positivity rate was $60 \%(270 / 453)$ and the 270 positive slides includes 245 P. falciparum, 18 P. vivax and seven $P$. malariae in December 2007 [18]. In comparison of the two active detections, the slide positivity was significantly decreased $\left(\mathrm{x}^{2}\right.$ =97.7836, $P<0.0001)$ while the ratios of $P$. falciparum versus $P$. vivax were not changed significantly $\left(\mathrm{x}^{2}=4.3554\right.$. $8, P=1.0369$ ). This shows that a high malaria burden was still present during the investigation. By 2013, the GFATM project decreased the API to 8.60 per 10,000 person-years (260/302496) thereby reducing the malaria burden dramatically in the whole SSR2. This success illustrates the importance of intervention coverage for malaria control $[8,17]$.

The analysis demonstrates that slide positivity was associated with age $(<15$ years), altitude $(<800 \mathrm{~m})$, lack of knowledge on malaria transmission and symptoms, the 
inaction of measures against mosquito bite and delayed treatment-seeking $(>48 \mathrm{~h}$ ). A retrospective case-control study documented that the Chinese migrants who stay overnight in the lowland, foothill and half hill areas, especially along the waterside in Myanmar, had higher risks of malaria infection [19]. The difference of slide positivity between male and female is not significant. However, children $(<15$ years) had a high slide positivity rate $(95.1 \%)$ in this study (Table 2). Also, a malaria (i.e. P. falciparum) outbreak in 2014 illustrated people aged $<15$ years had a high parasite prevalence rate $(84.4 \%)$ [10]. The demographic characteristics of parasite-positive individuals (children, no difference in sex) suggest that transmission was likely within the housing settlements and also gives evidence of higher than normal transmission in the region. After intervention, if the API is $<1$ per 1000 person-year, the program status can transfers from control to elimination, and from there infected cases are mainly imported, male and adult $[19,20]$. The findings above highlight the differences of demographic characteristics between endemic and elimination areas.

People's knowledge, awareness and behaviour may influence their action in self-protection and in seeking treatment, which further affects incidences of malaria infection and willingness to completion of treatment courses [12]. The results highlight that a lack of knowledge on malaria transmission and symptoms, the inaction of measures against mosquito bites and delayed treatment-seeking (> $48 \mathrm{~h}$ ) were independent risk factors of slide positivity. Decreasing sensitivity of anti-malarial drugs may be attributable to the delayed treatment-seeking and poor compliance to standard treatment course. The genetic diversity of malaria parasites and multiclonal infections are correlated with transmission intensity as well as the development and spread of anti-malarial resistance. High parasite population size and transmission intensity allow effective genetic recombination and mutation of malaria parasites [21, 22]. A sensitivity surveillance of Plasmodium falciparum to DAPQ in the two districts from 2007 to 2008 indicates that the fever clearance time (FCT) increased from $23.5 \pm 16.97 \mathrm{~h}$ in 2007 to $36.0 \pm 16.97 \mathrm{~h}$ in $2008(\mathrm{~F}=219.4, P<0.0001)$ and the asexual parasite clearance time (PCT) increased from $23.5 \pm 0.71 \mathrm{~h}$ in 2007 to $41.0 \pm 9.90 \mathrm{~h}$ in $2008(\mathrm{~F}=1485.4, \mathrm{P}<0.0001)$ [23]. The GFATM public health interventions improved local people's accessibility to qualified malaria prevention and treatment [8]. The FCT and PCT P. falciparum malaria cases were $36.4 \pm 8.9 \mathrm{~h}$ and $53.3 \pm 11.3 \mathrm{~h}$ in the 2014 outbreak [10]. The FCT has increased $0.4 \mathrm{~h}$ between 2008 and 2014 (6 years) but increased $2.5 \mathrm{~h}$ between 2007 and 2008 (1 year). The PCT is increased $12.1 \mathrm{~h}$ between 2008 and 2014 but increased $17.5 \mathrm{~h}$ between 2007 and 2008. The above differences indicate that effective interventions may slow down the declining sensitivity of $P$. falciparum to
DAPQ. As resistance of $P$. falciparum to several antimalarial drugs, including ACTs, has reached alarming levels in the GMS, there should be an emphasis on providing qualified treatment, a promotion of treatment-seek behaviour and compliance to cure treatment regimens [8].

Being of a young age might be associated with microscopically confirmed parasitemia because transmission in the children was sufficiently high to provide an increasingly robust immune response in adults. Results are almost certainly underestimated as they bias against those without fever. The true prevalence might be higher than estimated here and the magnitude of such underestimation is due to the prevalence of asymptomatic malaria in this population.

Finally, there remain two lessons from this study that may be of interest to following projects. First, the data used was from an investigation that ran from October 1st to December 31st, 2009. However, the findings obtained after reanalysing the old data and comparing it with the updated data are more meaningful. The analysis confirmed that malaria has not finally been controlled in the SSR2, especially in the Salween River Valley. We still need further investigation and more control of malaria foci in the GMS. Second, the study highly relies on the results of microscopy and a sample of self-report-fever villagers rather than other more reliable technics like molecular methods.

\section{Conclusion}

Independent risk factors for slide positivity are age, altitude, knowledge about malaria transmission and symptoms, self-protection against mosquito bites and treatment-seeking in the SRV of the SSR2. These five factors determined how likely the transmission of malaria was within the housing settlements and also helped researchers predict where higher than the normal transmission was likely to occur in the region. The reduced malaria burden of the SSR2 in recent years shows that promoting people's access to qualified treatment and promotion of treatment seeking behaviours are important for the malaria elimination and containment of artemisinin resistance.

\section{Abbreviations \\ 95\% Cl: Confidence interval; ACT: Artemisinin based therapy; AMl: Aide Medical International; AOR: Adjusted odds ratio; API: Annual parasitic incidences; CQ: Chloroquine; DAPQ: Dihydroartemisinin- piperaquine; FCT: Fever clearance time; GFATM: Global Fund to fight AIDS, Tuberculosis and Malaria; GMS: Greater Mekong Subregion; NGO: Nongovernment organization; OR: Odds ratio; PCT: Parasite clearance time; PQ: Primaquine; SRV: Salween River Valley; SSR2: Shan Special Region II; WHO: World Health Organization}

\section{Acknowledgements}

We thank Ms. Julie Watson and Mr. Hongzhang Xu from Fenner School of Environment and Society, Australian National University for their comments and copyediting language. We thank all participants for their contribution of time and patience in the study. 


\section{Funding}

The fifth grant to China of the Global Fund to fight AIDS, Tuberculosis and Malaria (GFATM) provided financial support for the field investigation in 2009. The data analysis and preparation of the manuscript were supported by the National Natural Science Foundation of China (No. 81560543 and 81673113).

\section{Availability of data and materials}

The datasets used and analyzed during the current study available from the corresponding author on reasonable request.

\section{Authors' contributions}

$H \mathrm{~L}, \mathrm{~J}-\mathrm{WX}$ and Q-ZX designed the study and developed the protocol, analyzed and interpreted the data. $\mathrm{HL}$ supervised the investigation and conducted microscopy in the field survey. Q-ZX, Y-R Z and HL conducted HHs survey. $\mathrm{HL}$ and J-WX wrote the first draft of the paper. All authors read and approved the final manuscript.

\section{Ethics approval and consent to participate}

The investigation was approved by Department of Health, Shan Special Region II, Myanmar. Ethical approval for the study under "The therapeutic efficacy study of anti-malarial drugs in the GMS" (Reference number: YIPDEC-2007[01]) was granted by the Ethics Committees of the Yunnan Institute of Parasitic Diseases, China. According to the World Medical Association Declaration of Helsinki, the purpose of the study was explained to the study participants. Informed written consent was obtained from all adult subjects or caretakers of children. All participants (adults and guardians for children) consent to report their data. However, all results were kept confidential and were unlinked to any identifying information.

\section{Competing interests}

The authors declare that they have no competing interests.

\section{Publisher's Note}

Springer Nature remains neutral with regard to jurisdictional claims in published maps and institutional affiliations.

\section{Author details}

YYunnan Institute of Parasitic Diseases, Yunnan Provincial Centre of Malaria Research, Yunnan Provincial Key Laboratory of Vector-borne Diseases Control and Research, Yunnan Provincial Collaborative Innovation Center for Public Health and Disease Prevention and Control, Puer 665000, China. 'People's Hospital of Taikang County, Taikang 475400, Henan Province, China.

${ }^{3}$ Mengmao County Hospital, Mengmao, Shan Special Region II, Myanmar.

Received: 20 September 2017 Accepted: 17 April 2018

Published online: 27 April 2018

\section{References}

1. WHO. Global technical strategy for malaria 2016-2030. Geneva: World Health Organization; 2015. Accessed 27 Feb 2016

2. WHO. World malaria report 2015. Geneva: World Health Organization; 2015. Accessed 24 May 2016

3. Cui L, Yan G, Sattabongkot J, Cao Y, Chen B, Chen X, et al. Malaria in the Greater Mekong Subregion: heterogeneity and complexity. Acta Trop. 2012; 121:227-39.

4. Delacollette C, Souza C, Christophel E, Thimasarn K, Abdur R, Bell D, et al. Malaria trends and challenges in the Greater Mekong Subregion. Southeast Asian J Trop Med Public Health. 2009;40:674-91.

5. Li N, Parker D, Yang Z, Fan Q, Zhou G, Ai G, Duan H, Lee M, Yan G, Matthews S, Cui L, Wang Y. Risk factors associated with slide positivity among febrile patients in a conflict zone of north-eastern Myanmar along the China-Myanmar border. Malar J. 2013;12:361.

6. WHO. Strategy for malaria elimination in the Greater Mekong Subregion: 20152030. Geneva: World Health Organization; 2015. Accessed 27 Feb 2016

7. Wang RB, Zhang J, Zhang QF. Malaria baseline survey in four special regions of northern Myanmar near China: a cross-sectional study. Malar J. 2014;13:302.

8. Xu JW, Li Y, Yang HL, Zhang J, Zhang ZX, Yang YM, et al. Malaria control along China-Myanmar border during 2007-2013: an integrated impact evaluation. Inf Dis Poverty. 2016;5:75.
9. $\mathrm{Xu} \mathrm{JW}$, Liu H. The relationship of malaria between Chinese side and Myanmar's five special regions along China-Myanmar border: a linear regression analysis. Malar J. 2016;15:368.

10. Liu H, Xu JW, Yang HL, Mei L, Sun CD, Yin YJ, et al. Investigation and control of a Plasmodium falciparum malaria outbreak in Shan Special Region II of Myanmar along the China-Myanmar border from June to December 2014. Inf Dis Poverty. 2016;5:32.

11. Dong XS. The malaria vectors and their ecology in Yunnan Province. Chin J Parasit D is. 2000:13:144-7. (in Chinese)

12. Xu JW, Xu QZ, Liu H, Zeng YR. Malaria treatment-seeking behaviour and related factors of Wa ethnic minority in Myanmar: a cross-sectional study. Malar J. 2012;11:417

13. Bureau for Disease control and prevention, Ministry of Health, People's Republic of China. Malaria control manual. Beijing: People's Health Publishing Company; 2007. p. 33-42. (in Chinese)

14. Liu H, Yang HL, Xu JW, Wang JZ, Nie RH, Li CF. Artemisinin-naphthoquine combination versus chloroquine-primaquine to treat vivax malaria: an openlabel randomized and non-inferiority trial in Yunnan Province, China. Malar J. 2013;12:409

15. Liu H, Xu J-W, Bi Y. Malaria burden and treatment targets in Kachin Special Region II, Myanmar from 2008 to 2016: A retrospective analysis. PLoS ONE. 2018:13:4.

16. $\mathrm{Xu} \mathrm{JW}$, Liu H. The challenges of malaria elimination in Yunnan Province, People's Republic of China. Southeast Asian J Trop Med Public Health. 2012; 43:819-24.

17. Xu JW, Li JJ, Guo HP, Pu SW, Li SM, Wang RH, Liu H, Wang WJ. Malaria from hyperendemicity to elimination in Hekou County on China-Vietnam border: an ecological study. Malar J. 2017;16:66.

18. Liu H, Nie RH, Li CF, Sun YH, Li GS. Active detection of malaria cases in Myanmar Wa ethnical villages of China-Myanmar border. Para Inf Dis. 2009; 12:6-8. (in Chinese)

19. Xu JW, Liu H, Zhang Y, Guo XR, Wang JZ. Risk factors for border malaria in a malaria elimination setting: a retrospective case-control study in Yunnan. China Am J Trop Med Hyg. 2015;92:546-51.

20. Cotter C, Sturrock HJ, Hsiang MS, Liu J, Phillips AA, Hwang J, et al. The changing epidemiology of malaria elimination: new strategies for new challenges. Lancet. 2013;382:900-16.

21. Yuan LL, Zhao H, Lanou Wu L, Li XM, Parkerd D, Xu SH, et al. Plasmodium falciparum populations from northeastern Myanmar display high levels of genetic diversity at multiple antigenic loci. Acta Trop. 2013;125:53-9.

22. Liu H, Yang HL, Tang LH, Li XL, Huang F, Wang JZ, et al. In vivo monitoring of dihydroartemisinin-piperaquine sensitivity in Plasmodium falciparum along the China-Myanmar border of Yunnan Province, China during 20072013. Malar J. 2015;14:47.

23. Liu H, Yang HL, Zhang J, Li CF, Nie RH, Wang HY. Clinical trial on efficacy of compound dihydroartemisinin-piperaquine for treatment of uncomplicated falciparum malaria in Myanmar. Chin J Parasit Parasitic Dis. 2011;29:296-8. (in Chinese)

Ready to submit your research? Choose BMC and benefit from:

- fast, convenient online submission

- thorough peer review by experienced researchers in your field

- rapid publication on acceptance

- support for research data, including large and complex data types

- gold Open Access which fosters wider collaboration and increased citations

- maximum visibility for your research: over $100 \mathrm{M}$ website views per year

At BMC, research is always in progress.

Learn more biomedcentral.com/submissions 